\title{
L'UTILISATION DE JEUX POUR L'ENSEIGNEMENT DE L'ANGLAIS COMME LANGUE SECONDE
}

\section{ARTICLE D'EXAMEN}

DIAS, Adailton Di Lauro ${ }^{1}$

DIAS, Adailton Di Lauro. L'utilisation de jeux pour enseigner l'anglais comme langue seconde. Revista Científica Multidisciplinar Núcleo do Conhecimento. Année 04, Ed. 07, vol. 03, pp. 69-76. juillet 2019. ISSN: 2448-0959

\section{ABSTRAIT}

Évaluant les pratiques d'enseignement actuelles et observant même les aspects historiquement méthodologiques, les élèves sont aujourd'hui confrontés à une difficulté extrême pour développer les compétences cognitives et acquérir des connaissances dans le système éducatif actuel. Peut-être que ce n'est pas dû à l'inefficacité des méthodes, mais à la dynamique des relations entre l'étudiant et l'environnement. Différents groupes d'âge exigent des approches différentes, qui ont changé à la condition de l'obsolescence; ils perdent leur efficacité de manière significative, au fil du temps. Ainsi, les jeux en termes d'âge et de sujets peuvent être un moyen efficace d'afficher le contenu en raison de leur universalité. Le présent article vise à expliquer les raisons pour lesquelles les jeux constituent un outil important dans l'éducation et à indiquer quelques façons d'appliquer ces outils dans le processus d'enseignement et d'apprentissage de l'anglais comme langue seconde.

Mots-clés: Jeu, capacité, langue, émotions, processus d'apprentissage.

\footnotetext{
1 Étudiant à la maîtrise en éducation scientifique à l'Université Unigrendal. Spécialiste en anglais par les collèges FIJ-Integrated de Jacarepagua, Rio de Janeiro (2007). Majoré en littérature par l'Université d'État de Bahia, Brésil. Professeur d'anglais et de portugais à l'IFRR-Brésil.
} 


\section{INTRODUCTION}

Considérant le progrès technologique à un rythme constant et, plus encore, en observant que les méthodologies devraient accompagner ces changements dans le but d'amélioration constante, cet article cherche à relier l'utilisation des jeux à la pratique de l'enseignement de l'anglais comme un langue seconde. L'étude ne se limite pas à un modèle spécifique du jeu, mais à toute façon ludique de simuler la réalité. Ainsi, l'analyse détaille certains types de jeux et ses aspects historiques en notant la relation étroite entre eux, le contexte et le processus d'apprentissage. La proposition actuelle peut converger vers une création de pratiques, qui utilisent des jeux pour rendre l'acte d'apprentissage agréable pour les élèves; il peut également les aider à utiliser le contenu proposé couramment et efficacement dans leur vie quotidienne.

L'absence de situations réelles qui contribuent à la pratique de la nouvelle langue par les étudiants constitue l'une des principales difficultés du processus d'enseignement de l'anglais. Dans l'environnement de la salle de classe, différentes activités peuvent combler une partie de cette lacune si l'enseignant développe l'intérêt des élèves pour l'apprentissage, en se concentrant sur les classes et les activités positives. Lorsque I'on considère les nombreuses possibilités dans un environnement où les élèves peuvent simuler des situations parascolaires et entrer dans le contexte de ces situations, un meilleur résultat peut être obtenu. Les perspectives d'ordre conceptuel dans cette étude tiennent compte de la littérature indiquée, mais surtout, considère l'expérience de l'auteur dans la pratique pédagogique, principalement dans l'enseignement de l'anglais comme langue seconde. Par conséquent, le point de vue et l'observation de l'auteur remplacent, dans cette étude, la recherche sur le terrain habituelle requise.

Basé sur des recherches de jeux pour enfants, jeux et autres concepts et sujets connexes, il existe différentes façons d'appliquer cette étude dans la sphère pédagogique. Pour Dantas (1998), « le terme ludique fait référence à l'acte de jouer (de manière libre et individuelle) et de jouer (en ce qui concerne une conduite sociale qui implique des règles) ». Cependant, la relation spécifique et dynamique entre la 
méthode et les élèves rend nécessaire l'approfondissement et la direction des jeux pour chaque matière. Selon ANTUNES (2003), pour rendre les jeux compatibles avec la pratique d'un enseignement efficace, il est nécessaire d'analyse et de recherche, car le résultat peut être inversé s'il n'y a pas de compatibilité entre eux et l'objet de l'apprentissage. La littérature utilisée guide une partie de la construction des concepts sans définir l'applicabilité des jeux. Ainsi, c'est l'auteur qui suggère une telle utilisation pratique et sa convergence méthodologique possible.

\section{QUELQUES CONSIDÉRATIONS SUR LES ÉTAPES DE L'APPRENTISSAGE}

Un point important à considérer est le contexte et les conditions, qui sont appliqués aux mécanismes d'apprentissage. En ce qui concerne les jeux, le public est très hétérogène, compte tenu de la variété des jeux existants et de la gamme illimitée liée à l'âge des élèves. Le jeu est une activité spontanée, effectuée par une ou plusieurs personnes et, différemment de l'art et le travail, peut être considéré comme un moyen de stimulation physique ou mentale, parfois les deux. L'existence des jeux remonte à l'époque préhistorique et couvre tous les niveaux d'expérience, les sexes et les âges. Parmi les étapes de l'apprentissage, il est important de comprendre que les obstacles à la survie sont la justification des différents processus. Ainsi, ces processus se produisent sur une échelle de complexité croissante. La création d'opérations nécessaires pour surmonter ces entraves, il passe par des phases différentes et bien définies, de l'enfance à l'adolescence.

La première étape commence à la naissance et dure généralement jusqu'au début de l'acquisition de la langue (jusqu'à environ dix-huit mois). Pendant cette période, la formation de la motricité et de la perception sensorielle commence. La deuxième étape se déroule environ entre deux et quatre ans et est définie par la formation de la pensée symbolique. L'utilisation de poupées, de voitures et d'autres objets de nature symbolique qui simulent la réalité, font partie de cette étape particulière. Entre quatre et huit ans, la troisième étape apparaît, où la pensée intuitive commence à se former et les objets autour deviennent les points de référence. 
Automatiquement, cette intuition conduit à la quatrième étape, qui est le stade où les humains apprennent à organiser des opérations concrètes pour associer tout objet référencé à son sens. La quatrième phase dure entre quatre et huit ans. La dernière étape est achevée pendant l'adolescence, où la construction des opérations mentales est suffisante pour la pensée analytique. En prenant ces mesures et leurs distinctions, il est possible de comprendre que la pertinence des jeux pour différents groupes d'âge peut rencontrer la plupart de leurs membres.

\section{USING THE GAMES AS A SUPPORT FOR TEACHING ENGLISH}

Pour WITTGENSTEIN (2001), le concept de "jeu" ne peut se limiter à une seule définition, mais comme une multiplicité de paramètres pour établir une relation de ressemblance familiale entre eux. La définition de la multiplicité de Wittgenstein trouve également un soutien dans CALLOIS (1957), ce qui donne quelques caractéristiques nécessaires pour qu'une activité soit définie comme un jeu. Les conditions sont que l'activité doit être amusante, limitée dans le temps et le lieu, de résultats imprévisibles et qui n'est pas productif. En outre, l'activité doit avoir des règles différentes de la vie quotidienne et doit être fictive, accompagnée de la conscience d'une réalité différente. Les jeux doivent donc approcher le réel de l'imaginaire et de la salle de classe, ou l'espace alternatif choisi doit être un environnement adapté à ces pratiques.

L'utilisation de la répétition dans les niveaux de langue, lors de l'enseignement de l'anglais, devrait se produire comme le processus d'enseignement de la langue maternelle, même pour les adultes débutants. À l'exception de l'apprentissage de la grammaire, la technique d'apprentissage de l'anglais avec l'index pointant vers des images ou des objets est très efficace. L'enseignant doit effectuer la présentation de la langue clairement et dans les jeux, vous devez éviter les activités qui conduisent à l'ennui ou la distraction. Au début, les tâches telles que la traduction et la grammaire ont très peu de mérite réel, puisque la langue écrite est secondaire à ce stade. L'objectif principal est de lier la langue au contexte et à l'environnement réel, abstrait ou fictif des jeux. 
Le choix des jeux doit être guidé par l'applicabilité au groupe d'âge en question, la faisabilité technique et l'état de suivi par l'enseignant à toutes les étapes de l'activité. Ainsi, les jeux éducatifs de construction ou d'instruction peuvent être employés puisqu'ils répondent aux conditions ci-dessus. Selon le but, l'un ou l'autre type de jeu peut être utilisé en fonction de diverses conditions, mais il est important de capter l'intérêt de toute la communauté impliquée. Voici quelques types de jeux définis qui peuvent être utilisés :

\subsection{JEUX INTERACTIFS ET INTERCHANGING}

Ces jeux sont utilisés pour rejoindre le membre d'un groupe. Ils aident les participants à mémoriser des informations spécifiques et à promouvoir une atmosphère détendue. Les joueurs sont distraits, vermifugés, libèrent les tensions et surmontent les réserves personnelles. Ils doivent être utilisés dans les premières phases du développement d'un groupe, lors du début d'une réunion, après une pause et chaque fois que le groupe semble commencer à se fatiguer, s'ennuyer ou pas motivé.

La musique et la danse sont recommandées dans les jeux actifs pour syntoniser le groupe dans l'activité proposée. La décision idéale est d'utiliser des jeux courts avec beaucoup d'action et une grande dépense d'énergie.

\subsection{JEUX DE TOUCHER ET DE CONFIANCE}

Ces jeux aident les participants à se regarder face à la confiance et la confiance dans leur vie. Selon l'organisation culturelle et le niveau d'ouverture des personnes, le groupe peut aller progressivement à d'autres exercices qui impliquent le toucher. Les jeux de toucher et de confiance doivent être utilisés avec beaucoup de soin; l'instructeur doit être attentif au moment et aux réactions du groupe ainsi qu'aux participants, en leur offrant des ressources pour bien faire face à de solides processus psychologiques internes. 


\subsection{JEUX DE CRÉATIVITÉ ET DE RÉFLEXION}

Ce sont des jeux qui stimulent l'expression imaginaire, l'intuition et la créativité. Dans ces jeux, les participants peuvent se remarquer et montrer ouvertement aux autres ce qu'ils ont découvert sur eux-mêmes, sur le sujet étudié et sur le groupe. Les joueurs entrent en contact avec leur intérieur et d'autres joueurs à l'intérieur, en remarquant ce qui est plus pertinent à tous les niveaux. Ce type de jeu doit être utilisé lorsque les groupes sont complètement intégrés, travaillant ensemble et en utilisant toutes les conditions pour aller profondément dans le sujet étudié jusqu'à ce moment.

\subsection{JEUX DE GESTION}

Ces jeux concentrent l'attention des joueurs sur la planification, la gestion des ressources, la simulation de situations et l'apprentissage de techniques spécifiques. Ce type de jeu doit être utilisé après que le groupe est bien intégré afin qu'ils puissent atteindre l'objectif proposé. Parfois, il est courant d'apparaître quelques difficultés dans l'apprentissage du sujet il est donc très important que tout le cycle d'apprentissage vivant est travaillé complètement.

\subsection{JEUX DE CLÔTURE}

Ces jeux aident les gens à avoir la chance de définir leur position liée à un sujet, au groupe et à eux-mêmes, en transférant ce qu'ils ont obtenu au cours du processus d'apprentissage à leur vie quotidienne. Les jeux de synthèse et de fin sont ceux qui ritualisent, évaluent et formalisent ce qui a été fait pendant le travail. II est vraiment important de préciser, pour chaque participant, qu'un cycle est en train de se terminer et qu'un autre commence.

\subsection{JEUX D'ADRESSE ET DE STRATÉGIE}

Peut être utilisé à n'importe quelle étape du processus ou des situations. II est important de souligner que le sport est inclus dans presque toutes les modalités expliquées ci-dessus, mais il doit être utilisé afin d'impliquer le groupe sur l'aspect de 
l'apprentissage, mais sans abandonner l'aspect compétitif, qui est précisément ce qui attire le public de groupes d'âge. Le jeu, organisé comme une activité de plaisir, se constitue comme un outil à étudier et à appliquer à l'enseignement de l'anglais principalement parce qu'il est connu que notre cerveau apprend par un processus de répétition, d'essai et de vitesse (VILLA - SANTANDER, 2003). L'essai, ou l'expérimentation, est l'analyse de la situation. La répétition donne la pratique qui mène à la vitesse, ou l'efficacité et l'efficacité. La capacité de chaque élève indiquera le niveau de jeu à utiliser.

Comprenant, par conséquent, les différentes définitions des jeux et la question des principes de l'apprentissage de l'homme, il devient clair la condition de leur pleine utilisation dans les pratiques d'enseignement. L'intérêt de l'étudiant pour l'apprentissage est lié à son intérêt pour le jeu proposé à l'époque. L'environnement créé par le jeu, en tenant compte aussi du fait que les émotions qui influencent les êtres humains favorisent également l'assimilation, peut fournir l'absence de situations réelles. Ainsi, plus l'excitation causée dans les jeux est élevée, plus la possibilité de définir le contenu proposé est grande.

Les émotions positives favorisent plus d'apprentissage (SISTO et MARTINELLI, 2006), de sorte que le sentiment de joie surmonte la tristesse ou la douleur. L'enseignant, quant à lui, commence à jouer le rôle de médiateur entre le réel et l'imagination représentée par le jeu et son résultat. La salle de classe passe d'un lieu de discours pédagogique à un laboratoire de partage d'expériences, plein de possibilités.

\section{CONCLUSION}

Cette étude a tenté d'offrir une alternative au processus d'enseignement de l'anglais comme langue seconde, à travers des jeux, en cherchant à agréger les valeurs au contexte éducatif actuel. Enseigner une langue, hors de son environnement naturel, est un processus qui exige des compétences et de la créativité de la part des professionnels concernés. Puis, à partir du moment de choisir les jeux à l'analyse des résultats, l'enseignant doit prendre soin de suivre l'évolution de la reconnaissance des 
élèves, afin de ne pas perdre le véritable objectif. L'objectif est non seulement d'élaborer des stratégies, mais aussi de les faire fonctionner correctement.

Il est certain que la question ne s'arrête pas là. L'étude constante et le partage des expériences continuent d'être la responsabilité des personnes impliquées dans le processus d'enseignement et d'apprentissage. L'utilisation et la diffusion plus ou moins importantes des procédures où les pratiques suggérées sont utilisées détermineront leur succès. Considérant l'espace éducatif comme quelque chose à améliorer et, selon son dynamisme, ne peut pas tenir les concepts statiques et obsolètes.

\section{RÉFÉRENCES}

ABERASTURY, A. A criança e seus jogos. Porto Alegre: Artes Médicas, 1992.

AFFONSO, R. M. Ludodiagnóstico. São Paulo: Plêiade, 1995.

AGUIAR, J. S. Jogos para o ensino de conceitos: leitura e escrita na pré-escola. Campinas: Papirus, 1998.

ANTUNES, C. Jogos para a estimulação das múltiplas inteligências. $12^{a}$ edição. Petrópolis, RJ: Vozes, 2003.

CALLOIS, Roger. Les Jeux et les hommes. Paris: Gallimard, 1957.

DANTAS, H. Brincar e Trabalhar. In: KISHIMOTO, T. M. (org). Brincar e suas teorias. São Paulo: Pioneira, 1998.

SISTO, F. F. \& MARTINELLI, S. de C. (orgs.) Afetividade e dificuldades de aprendizagem: uma abordagem psicoeducacional. São Paulo: Vetor, 2006.

VILA, Magda \& SANTANDER, Marli. Jogos cooperativos no processo de aprendizagem acelerada. São Paulo: Qualitymark, 2003

WITTGENSTEIN, Philosophical Investigations, Blackwell Publishing Ltd., MA: 2001. 
Soumis : juin 2019..

Aprovate: Juillet, 2019. 